\title{
Die Politik der Troika am Beispiel Griechenlands
}

\author{
von Matthias Mors und Stefan Appel ${ }^{1}$
}

Dieser Aufsatz versucht am Beispiels Griechenland zu erklären, vor welchen Herausforderungen die sogenannte „Troika“ - Europäische Kommission, Europäische Zentralbank (EZB) und Internationaler Währungsfonds (IWF) - bei der Gestaltung und Überprüfung wirtschaftlicher Anpassungsprogramme für Mitgliedstaaten der Eurozone gestanden hat und wie die Troika die Situation gemeistert hat. Nach einem kurzen Rückblick auf die Krise wird die wirtschaftliche Entwicklung Griechenlands in den letzten Jahren analysiert, wobei die bisher erzielten Erfolge, aber auch die Kritikpunkte des Anpassungsprozesses beleuchtet werden. Insgesamt gesehen, kann die Zusammenarbeit innerhalb der Troika im Rahmen der Anpassungsprogramme für Griechenland, Irland, Portugal und Zypern als erfolgreich angesehen werden. Die Zusammenführung der jeweiligen Erfahrung und Expertise der drei Troika-Institutionen ist der Qualität der Analyse und der Programmempfehlungen zugutegekommen, auch wenn sie einen höheren Koordinierungsbedarf impliziert. Es ist jedoch einzuräumen, dass gelegentlich Meinungsunterschiede in einigen strategischen Punkten zwischen den drei beteiligten Institutionen die Zusammenarbeit zeitweise erschwert und zum Teil langwierige Diskussionen auf politischer Ebene erfordert haben.

By looking at the example of Greece, this essay attempts to explain the particular challenges the so-called Troika - European Commission, European Central Bank (ECB), and International Monetary Fund (IMF) - were confronted with in designing and monitoring economic adjustment programmes for euro area Member States and how the situation has been mastered. After looking back at the crisis, the economic development of Greece is analysed, shedding light on the success as well as the points of criticism experienced so far. Overall, the cooperation of the Troika in the context of the adjustment programmes for Greece, Ireland, Portugal and Cyprus can be regarded as a success. The joint experience and expertise of the three Troika institutions helped to improve the quality of analysis and programme recommendations even if it implied higher coordination needs. This notwithstanding, occasional differences between the Troika partners as regards a few strategic issues made the cooperation at times difficult and sometimes required difficult discussions at political level.

1 Die in diesem Artikel vertretenen Ansichten sind diejenigen der Autoren und nicht notwendigerweise diejenigen der Europäischen Kommission. 


\section{Einführung}

Als die Eckpfeiler der Europäischen Wirtschafts- und Währungsunion in den 90er Jahren konzipiert wurden, galt es als ausgeschlossen, dass ein Euroland in dramatische finanzielle Schwierigkeiten geraten könnte und finanzielle Hilfe von außen benötigen würde. Zwar waren einige Nicht-Euroländer von den Auswirkungen der Finanzkrise in den Jahren 2008/2009 derartig betroffen, dass sie externe finanzielle Unterstützung zur Lösung ihrer Zahlungsbilanzprobleme benötigten (Ungarn, Lettland und Rumänien), aber dies schien die Eurozone nicht zu betreffen. Im April 2010 fragte Griechenland jedoch als erstes Euroland um Hilfe, auch wenn diese Anfrage hauptsächlich durch inländische Faktoren und nicht durch die internationale Finanzkrise ausgelöst wurde. Im Verlauf der Krise wurde jedoch die finanzielle und wirtschaftliche Situation auch einiger anderer Euroländer zunehmend prekärer. Der Lauf der Ereignisse hat gezeigt, wie schwer sich die Politiker in Griechenland wie auch in den Geberländer getan haben, die tatsächliche finanzielle und wirtschaftliche Lage Griechenlands zu akzeptieren und entsprechende Hilfen letztlich zuzusagen. ${ }^{2}$

Das griechische Programm hat die Schlagzeilen und Emotionen in vielen EU Mitgliedsstaaten in den letzten Jahren bestimmt, nicht zuletzt in Deutschland, da es alle Ingredienzien eines politischen und ökonomischen Dramas aufgewiesen hat: Die gewaltige Größenordnung der benötigten Hilfe, wiederkehrende Probleme bei der Programmimplementierung, die Notwendigkeit einer erheblichen Aufstockung der Hilfe in einem zweiten Programm, mehrmalige Datenrevisionen, einen in seiner Größenordnung bisher einmaligen Schuldenschnitt, erratische öffentliche Kommunikation von Schlüsselrepräsentanten auf Geber- und Nehmerseite, das Scheitern der Regierung und Neuwahlen, das Aufdecken von in einem EU Mitgliedsland ungeahnten administrativen und strukturellen Problemen und Mängeln, etc. Zudem hat es die Glaubwürdigkeit des Landes deutlich erschüttert, dass Griechenland - wie revidierte Statistiken belegen - die Maastricht-Kriterien zum Eintritt in die Eurozone in Wahrheit nie erfüllt hatte.

All dies hat die politische und mediale Diskussion der Probleme Griechenlands in der Öffentlichkeit aufgeheizt und emotionalisiert. Alle Anpassungsprogramme, aber insbesondere im Falle Griechenlands, haben in vielen Geberländern zu

2 European Commission: The economic adjustment Programme for Greece, European Economy, Occasional Paper No. 61, Mai 2010, abrufbar unter: http://ec.europa.eu/economy_finance/publications/ occasional_paper/2010/pdf/ocp61_en.pdf. Der Kommissionsbericht gibt eine detaillierte Beschreibung der finanziellen und wirtschaftlichen Ausgangsposition Griechenlands. Siehe insbesondere S. 8 für eine Auflistung wichtiger Ereignisse. 
der Entstehung radikalerer Parteien geführt (so wie die Haushaltskonsolidierungspolitik auch zu einer Ausfächerung und Radikalisierung der politischen Landschaft in den Schuldnerstaaten geführt hat).

Dieser Aufsatz versucht am Beispiel Griechenlands zu erklären, vor welchen Herausforderungen die sogenannte „Troika“ - Europäische Kommission, Europäische Zentralbank (EZB) und Internationaler Währungsfonds (IWF) - bei der Programmgestaltung und -überprüfung gestanden hat und wie die Troika die Situation gemeistert hat. Nach einem kurzen Rückblick auf die Krise wird die wirtschaftliche Entwicklung Griechenlands in den letzten Jahren analysiert. Anschließend wird die Zusammenarbeit der Troikapartner im Falle Griechenlands kritisch beleuchtet.

\section{Ein kurzer Rückblick}

\section{Griechenland nach Eintritt in die Wirtschafts- und Währungsunion}

Seit der Einführung des Euro in Griechenland am 1. Januar 2001 hatte Griechenland ein vordergründig gesundes Wirtschaftswachstum aufzuweisen. In den Jahren 2001-2008 erreichte es jährliche Wachstumsraten des realen Bruttoinlandsprodukts (BIP) von knapp $4 \%$ (Abb. 1), fast doppelt so hoch wie im Durchschnitt der Eurozone. Dadurch gelang es, das durchschnittliche pro-Kopf Einkommen in Griechenland von unter $75 \%$ des EU-Durchschnitts im Jahre 2000 auf rund $88 \%$ im Jahre 2009 zu steigern (Abb. 2). Oberflächlich gesehen schien Griechenland also bis zum Ausbruch der internationalen Finanzkrise ein Beispiel für den erfolgreichen Aufholprozess der ärmeren Mitgliedsländer in der EU zu stehen. Auch die Finanzmärkte schienen keine Zweifel am griechischen Wirtschaftsmodell zu haben, was sich in historisch niedrigen Zinsen ausdrückte (Abb. 3). 
Abbildung 1: Wachstumsrate des realen BIP

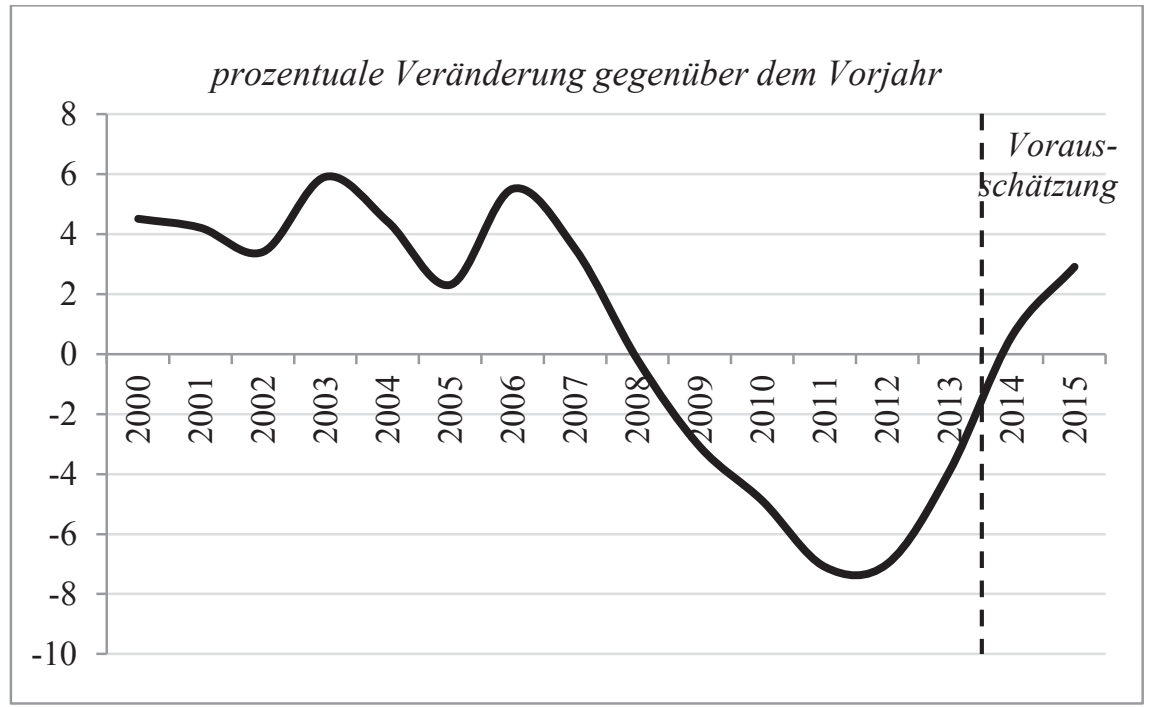

Quelle: Eurostat, Kommissionsdienststellen

Abbildung 2: Pro-Kopf BIP (KKS) im Vergleich zum EU-Durchschnitt

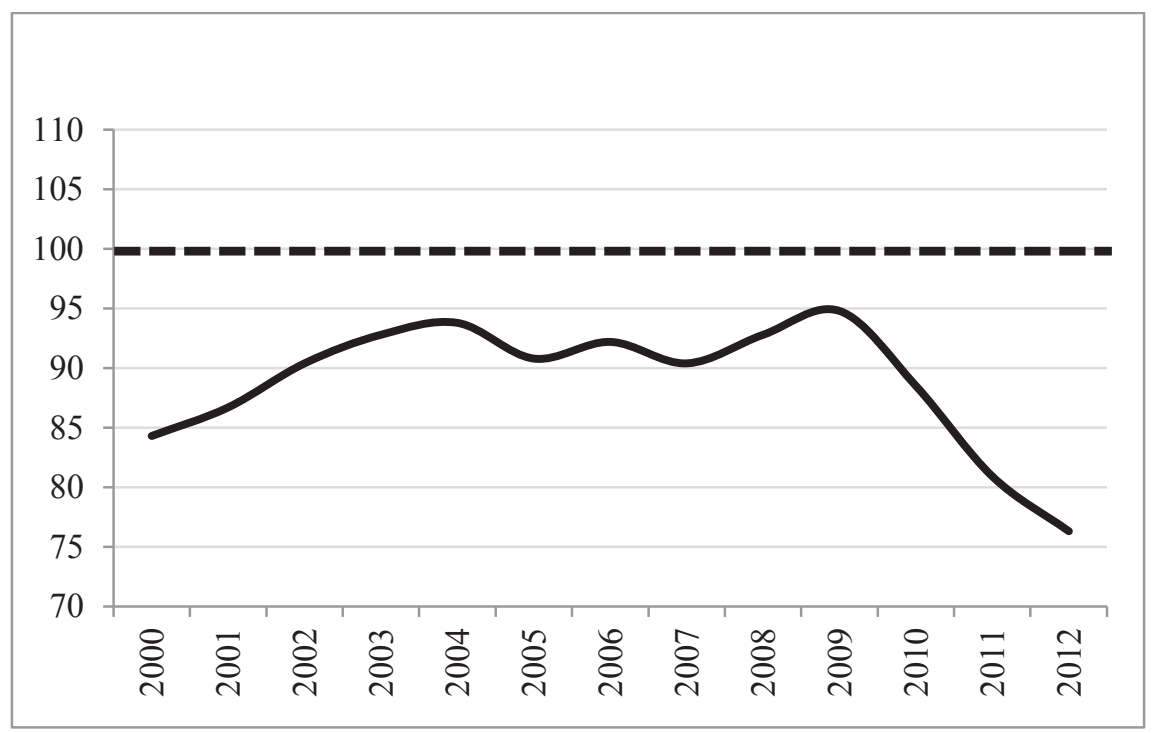

Quelle: Eurostat 
Abbildung 3: Zinssatz auf 10-jährige Staatsanleihen

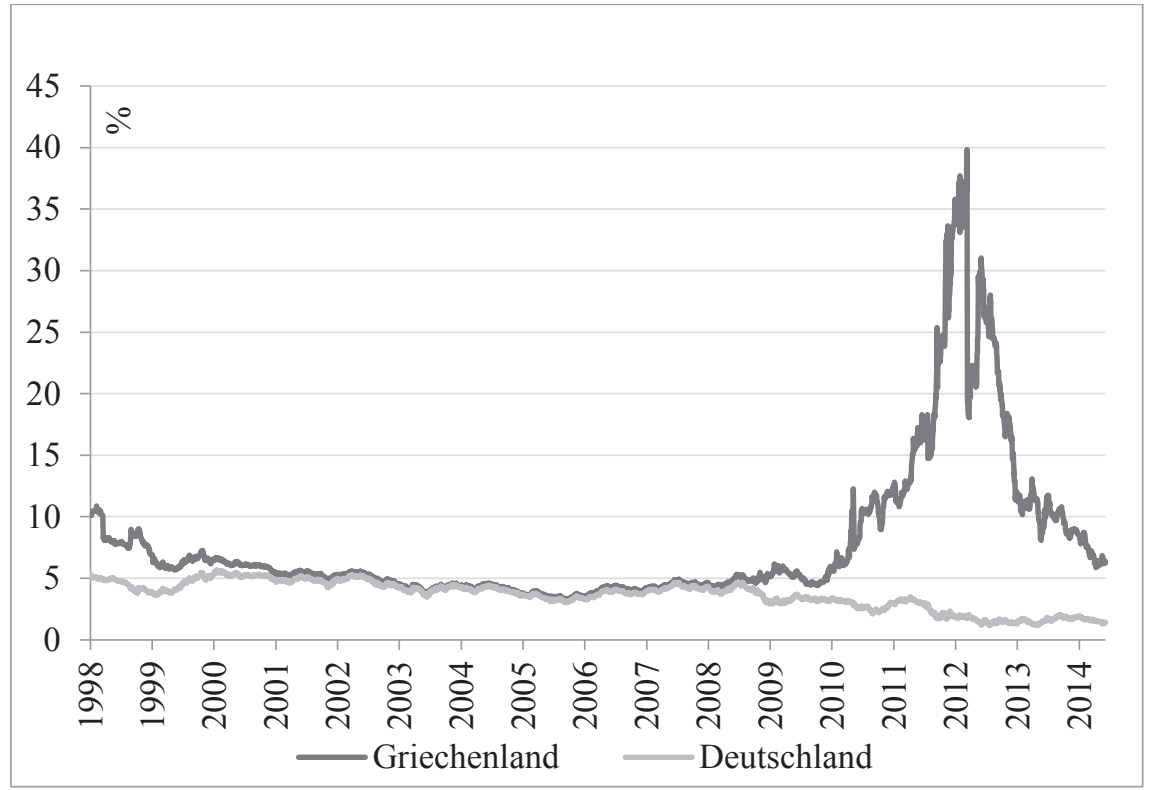

Quelle: ThomsonReuters

Das Wachstum in Griechenland beruhte jedoch hauptsächlich auf einem durch umfangreiche Kapitalzuflüsse gespeisten starken Wachstum des privaten und staatlichen Verbrauchs und, angeregt durch die historisch niedrigen Zinsen, des Wohnbaus. Zudem verfolgte Griechenland in den Jahren vor Ausbruch der Krise eine stark expansive Fiskalpolitik. Gleichzeitig wuchsen die Reallöhne deutlich stärker als die Produktivität, stimuliert auch durch starke Lohnsteigerungen im öffentlichen Dienst, sodass sich die internationale Wettbewerbsfähigkeit der griechischen Unternehmen deutlich verschlechterte. ${ }^{3}$ Der Boom des privaten Verbrauchs und die verschlechterte Wettbewerbsfähigkeit schlugen sich auch in einer sich rasch verschlechterten Außenhandelsbilanz nieder. Im Jahre 2008, erreichte das Leistungsbilanzdefizit die Rekordmarke von $14 \%$ des BIP (Abb. $4)$.

3 Siehe zu diesem Thema auch El-Shagi, M./ Lindner, A./ von Schweinitz, G.: Geriet die preisliche Wettbewerbsfähigkeit von Euroraum Ländern nach Gründung der Währungsunion aus dem Gleichgewicht?, in: Wirtschaft im Wandel, IWH, Jg. 20 (3), (2014), 46-49. 
Abbildung 4: Leistungsbilanzsaldo ( $\%$ des BIP)

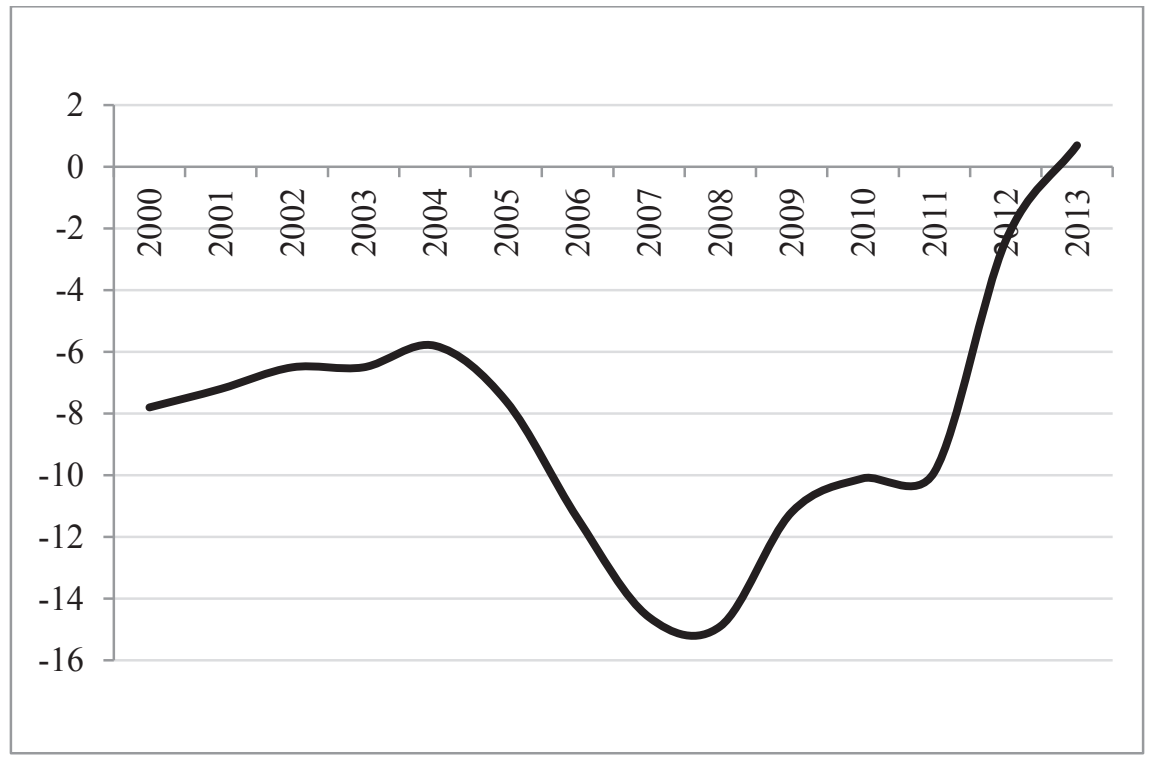

Quelle: Eurostat

\section{Der Ausbruch der Schuldenkrise in der Eurozone}

Die globale Finanz- und Wirtschaftskrise hatte direkte und indirekte Auswirkungen auf Europa. Während viele Probleme in Eurozonenländern hausgemacht waren, haben der Kollaps der amerikanischen Investmentbank Lehman Brothers im September 2008 und die dramatischen Folgen auf den Finanzmärkten weltweit die Krise auch in Europa verstärkt. Von einer Finanzkrise hat sich die Krise in der EU in kurzer Zeit in eine Wirtschaftskrise und anschließend in eine Schuldenkrise weiterentwickelt.

Das erste griechische Anpassungsprogramm vom Mai 2010 machte den Anfang einer Reihe ähnlicher „Troika-Programme“ für Mitgliedstaaten der Eurozone (siehe Tabelle 1). Im Dezember 2010 folgte das Programm für Irland und im Mai 2011 das Programm für Portugal. Nach dem zweiten griechischen Anpassungsprogramm im März 2012 kam im Mai 2013 Zypern hinzu. Inzwischen sind die Programme für Irland und Portugal erfolgreich abgeschlossen. Tabelle 1 veranschaulicht auch die schrittweise Weiterentwicklung der Finanzierungsinstrumente auf europäischer Seite. 
Tabelle 1: Troika-geführte Anpassungsprogramme für Mitgliedstatten der Eurozone

\begin{tabular}{|c|c|c|c|c|}
\hline & $\begin{array}{l}\text { Griechenland } \\
\text { I und II }\end{array}$ & Irland & Portugal & Zypern \\
\hline Е & $\begin{array}{l}\text { Mai 2010-Dezember } \\
2014 \\
\text { (IWF: Q1 2016) }\end{array}$ & $\begin{array}{l}\text { Dezember 2010- } \\
\text { Dezember } 2013\end{array}$ & $\begin{array}{l}\text { Mai 2011-Mai } \\
2014\end{array}$ & $\begin{array}{l}\text { Mai 2013-März } \\
2016\end{array}$ \\
\hline 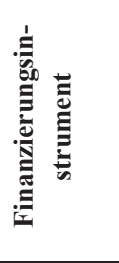 & $\begin{array}{l}\text { I: Bilaterale Darlehen } \\
\text { EU (GLF), IWF } \\
\text { II: EFSF, IWF* }\end{array}$ & $\begin{array}{l}\text { EFSF, EFSM, } \\
\text { IWF*, bilaterale } \\
\text { Darlehen von } \\
\text { Vereinigtem } \\
\text { Königreich, } \\
\text { Schweden, } \\
\text { Dänemark }\end{array}$ & $\begin{array}{l}\text { EFSF, EFSM, } \\
\text { IWF* }\end{array}$ & ESM, IWF* \\
\hline 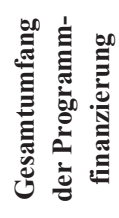 & $\begin{array}{l}\text { I: } 110 \text { Mrd. } € \text { (davon } \\
\text { 30 Mrd. } € \text { IWF) } \\
\text { II: } 130 \text { Mrd. } € \text { (davon } \\
\text { 9,8 Mrd. } € \text { IWF bis } \\
\text { Q4 2014) }\end{array}$ & $\begin{array}{l}85 \text { Mrd. } € \text { (davon } \\
22,5 \text { Mrd. } € \text { IWF } \\
\text { und 17,5 Mrd } \\
\text { Nationaler Pensi- } \\
\text { onsfonds) }\end{array}$ & $\begin{array}{l}78 \text { Mrd. } € \text { (davon } \\
26 \mathrm{Mrd} . € \mathrm{IWF})\end{array}$ & $\begin{array}{l}10 \text { Mrd. } € \text { (davon } \\
1 \mathrm{Mrd} . € \mathrm{IWF})\end{array}$ \\
\hline 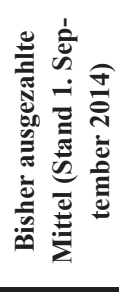 & $\begin{array}{l}\text { I: } 75,6 \text { Mrd. } €^{4} \\
\text { II: } 141,8 \text { Mrd. } € \\
\text { EFSF (davon } 24 \text { Mrd. } \\
€ \text { Transfer von I) und } \\
12 \text { Mrd. } € \text { IMF } \\
\text { (davon } 10 \text { Mrd } € \\
\text { Transfer von I) }\end{array}$ & 85 Mrd. $€$ & 76 Mrd. $€$ & 5,8 Mrd. $€$ \\
\hline : & $\begin{array}{l}\text { 1. Haushaltskonsoli- } \\
\text { dierung } \\
\text { 2. Haushaltskontrolle } \\
\text { 3. Wettbewerbsfä- } \\
\text { higkeit } \\
\text { 4. Finanzstabilität }\end{array}$ & $\begin{array}{l}\text { 1. Finanzstabili- } \\
\text { tät } \\
\text { 2. Haushaltskon- } \\
\text { solidierung } \\
\text { 3. Wettbewerbs- } \\
\text { fähigkeit }\end{array}$ & $\begin{array}{l}\text { 1. Haushaltskon- } \\
\text { solidierung } \\
\text { 2. Wettbewerbs- } \\
\text { fähigkeit } \\
\text { 3. Finanzstabili- } \\
\text { tät }\end{array}$ & $\begin{array}{l}\text { 1. Finanzstabili- } \\
\text { tät } \\
\text { 2. Haushaltskon- } \\
\text { solidierung } \\
\text { 3. Wettbewerbs- } \\
\text { fähigkeit }\end{array}$ \\
\hline
\end{tabular}

* Die vom IWF zu Programmbeginn zugesagten Beträge (in SDR) können wegen $€ / S D R$ Wechselkursschwankungen von den im Programmverlauf tatsächlich ausgezahlten Beträge abweichen.

4 Nicht ausgezahlte Mittel von insgesamt 34,4 Mrd $€$ (davon 10 Mrd $€$ IWF) wurden auf das zweite Programm übertragen. 


\section{Die Entscheidung für ein griechisches Anpassungsprogramm}

Die außergewöhnlich günstigen weltwirtschaftlichen Daten vor Ausbruch der Finanzkrise mit umfangreichen Kapitalzuflüssen in periphären Mitgliedstaaten zu historisch niedrigen Zinssätzen, hatten die tiefgreifenden Strukturprobleme Griechenlands weitgehend überdeckt. Zu Beginn des Jahres 2010 verschlechterte sich die Lage deutlich. Die von der neuen Regierung Papandreou bekanntgemachte Explosion des Budgetdefizites unter der Vorgängerregierung verunsicherte die Investoren und war der Auslöser dafür, dass Griechenland zunehmend Schwierigkeiten hatte, sich am Markt zu finanzieren. Innerhalb weniger Wochen schossen die Zinsdifferentiale zwischen deutschen und griechischen 2Jahresanleihen von rund 340 Basispunkten auf über 1550 Basispunkte Ende April. Angesichts dieser dramatischen Entwicklung bat die griechische Regierung am 23. April 2010 bei der Eurozone und dem IWF um Finanzhilfe.

Die griechische Anfrage traf die Eurozone in vieler Hinsicht unvorbereitet. Zwar hatte die Europäische Union in den Jahren davor schon Zahlungsbilanzhilfsprogramme für Ungarn, Rumänien und Lettland aufgestellt, aber die dafür verwendete Rechtsgrundlage war für Mitgliedstaaten der Eurozone nicht anwendbar. Es war daher notwendig zu improvisieren und bilaterale Darlehen der Mitgliedstaaten der Eurozone in der sogenannten „Greek Loan Facility“ zu bündeln. Dies geschah jedoch erst nach schwierigen Diskussionen über die Vereinbarkeit einer derartigen Hilfe mit dem im Vertrag über die Arbeitsweise der Europäischen Union niedergelegten Verbot einer Haftung, innerhalb der Währungsunion, für die Verbindlichkeiten eines anderen Mitgliedstaates sowie über die Bedingungen einer derartigen Hilfe (z.B. den Zinssatz).

Der Finanzbedarf des ersten Programms wurde auf 110 Mrd. Euro geschätzt, wobei 80 Mrd. von den anderen Mitgliedstatten der Eurozone (außer der Slowakei) und 30 Mrd. vom IWF im Rahmen eines „Stand-by Agreement“ getragen werden sollten. Im Laufe des Jahres 2011 setzte sich jedoch die Erkenntnis durch, dass die Finanzmittel nicht ausreichen würden. Im März 2012 wurde daher ein zweites Programm im Umfang von insgesamt 130 Mrd. Euro ins Leben gerufen und der IWF wechselte zu einem Programm auf Grundlage der „Extended Fund Facility“, was eine längere Rückzahlfrist vorsieht. Insgesamt wurden somit von öffentlichen Gläubigern Mittel von ursprünglich $240 \mathrm{Mrd}$. Euro für den Zeitraum 2010-2014 zugesagt. 


\section{Die Schwerpunkte des griechischen Anpassungsprogramms}

\section{Haushaltskonsolidierung und fiskalische Reformen}

Während Griechenland zum Zeitpunkt der Entscheidung zur Einführung des Euro im Jahre 2000 entsprechend den damaligen Statistiken nur ein Haushaltsdefizit von $2 \%$ aufwies, verschlechtere sich die Lage in den darauffolgenden Jahren kontinuierlich (Abb. 5), sodass die Europäische Kommission 2004 dem Rat die Eröffnung eines Verfahrens hinsichtlich eines übermäßigen Haushaltsdefizits vorschlug. Dieses Verfahren wurde im Juni 2007 nach Abbau des übermäßigen Defizits wieder geschlossen. ${ }^{5}$ Danach verschlechterte sich die Haushaltslage jedoch wieder dramatisch, sodass im April 2009 ein neues Verfahren eröffnet wurde.

Die rasante Verschlechterung innerhalb kürzester Zeit lässt sich plastisch anhand einiger Zahlen verdeutlichen. Während der griechische Haushalt für 2009 nur ein Defizit von $2 \%$ vorsah, wurde dieser Wert in dem nach dem Stabilitäts- und Wachstumspakt jährlich vorzulegenden Stabilitätsprogramm vom Januar 2009 bereits auf 3,9 \% nach oben revidiert. Im Oktober des gleichen Jahres meldete die neue griechische Regierung jedoch ein Defizit für das laufende Jahr von 12,7 \% und im April 2010 von 13,7 \%. Diese Explosion des Haushaltsdefizits war nicht nur der rapiden Verschlechterung der Haushaltslage zuzuschreiben, sondern reflektierte auch gravierende Probleme bei der Haushaltskontrolle und den Haushaltsstatistiken. Bei einer anschließenden Überprüfung der statistischen Zahlen durch die neue Regierung in Zusammenarbeit mit Eurostat wurde das Haushaltsdefizit nachträglich noch auf historische 15,6\% des BIP nach oben revidiert.

$\mathrm{Zu}$ Beginn des Jahres 2010 bei der Ausarbeitung des Anpassungsprogrammes war die Haushaltssanierung daher unvermeidlich einer der Hauptbestandteile des Programmes. Dabei war von Anfang an klar, dass sowohl auf der Ausgabenseite als auch auf der Einnahmenseite weitreichende Konsolidierungsmaßnahmen nötig wären. Um die Glaubwürdigkeit des Anpassungsprogramms zu stärken, sollte der Schwerpunkt der Konsolidierungsbemühungen auf der Ausgabenseite und auf Maßnahmen mit relativ geringem Umsetzungsrisiko liegen. Schließlich waren die Ausgaben des Staates in nur vier Jahren zwischen 2005-2009 um fast zehn Prozentpunkte des BIP von etwas über $44 \%$ auf $54 \%$ des BIP explodiert

5 Nachträglich stellte sich heraus, dass diese Entscheidung auf inkorrekten statistischen Zahlen beruhte und dass das Haushaltsdefizit sowohl bei Einführung des Euros als auch im Jahre 2007 tatsächlich nicht unter die 3-Prozentmarke gefallen war. 
(Durchschnitt der Eurozone: $51 \%$ ) während die Einnahmen sogar leicht auf rund $38 \%$ zurückgegangen sind (Durchschnitt der Eurozone $45 \%$ ). ${ }^{6}$ Allein drei Prozentpunkte dieses Ausgabenanstiegs waren auf zusätzliche Pensionsausgaben und über vier Prozentpunkte auf höhere Lohnkosten im öffentlichen Dienst zurückzuführen.

Das Paket von Konsolidierungsmaßnahmen bestand hauptsächlich aus strukturellen Maßnahmen und war stark auf eine rasche Anpassung angelegt. Erfahrungen in anderen Mitgliedstaaten (z.B. Lettland) hatten gezeigt, dass eine rasche, ausgabenorientierte Strategie die Anpassungskosten verringern kann. Allein im Jahr 2010 wurden Konsolidierungsmaßnahmen im Umfang von rund $8 \%$ des BIP ergriffen. Aufgrund des starken Rückgangs des BIP musste Griechenland im Laufe der Jahre jedoch Konsolidierungsmaßnahmen in viel höherem Umfang umsetzen als es sich in der Verbesserung des Haushaltsdefizits niederschlägt, da es gleichzeitig die mit der tiefen Rezession verbundene Verschlechterung der Haushaltslage (automatische Stabilisatoren) zu kompensieren hatte. Die Verbesserung des strukturellen Defizits um rund 17 Prozentpunkte im Zeitraum 20102014 unterschätzt also deutlich den Umfang der ergriffenen Konsolidierungsmaßnahmen.

Ausgabenwirksame Strukturreformen waren von Anfang an ein Schwerpunkt des Anpassungsprogramms. Dies betraf neben dem Aufbau einer soliden Ausgabenplanung und -kontrolle besonders die Reform der öffentlichen Verwaltung, den Bereich der Renten- und Sozialausgaben sowie den Gesundheitssektor. Analysiert man die Kürzungen auf der Ausgabenseite im Detail, so lässt sich feststellen, dass insbesondere beim staatlichen Verbrauch (z.B. Verteidigungsausgaben; Gesundheitssystem), bei den Löhnen und Pensionen im öffentlichen Dienst und bei den öffentlichen Investitionen (1\% des BIP) gespart wurde.

Auf der Einnahmenseite hatte Griechenland mit dem Problem eines ineffizienten Steuersystems und einer völlig dysfunktionalen Steuerverwaltung zu kämpfen. Neben strukturellen Maßnahmen zum Aufbau einer effizienten Steuerverwaltung wurden sowohl die direkten als auch die indirekten Steuern deutlich erhöht (Eigentumssteuern um rund $2 \%$ des BIP, Einkommensteuer, Mehrwertsteuer, Akzisen).

6 Für eine detaillierte Analyse der langfristigen Ausgabentrends siehe Kollintzas, T./ Papageorgiou, D./ Vassilatos, $V$ : An Explanation of the Greek Crisis: The Insiders - Outsiders Society, CEPR Discussion Paper No. 8996, 2012. 
Bei den Konsolidierungsmaßnahmen, sowohl auf der Ausgaben- wie auf der Einnahmenseite wurde im griechischen Anpassungsprogramm in der Regel versucht, die Lastenverteilung progressiv zu gestalten. Die Bemühungen um eine sozial ausgewogene Haushaltskonsolidierung wurden jedoch durch den mit der tiefen Rezession verbundenen Rückgang des Volkseinkommens sehr erschwert. Abbildung 5: Einnahmen und Ausgaben des Gesamtstaates

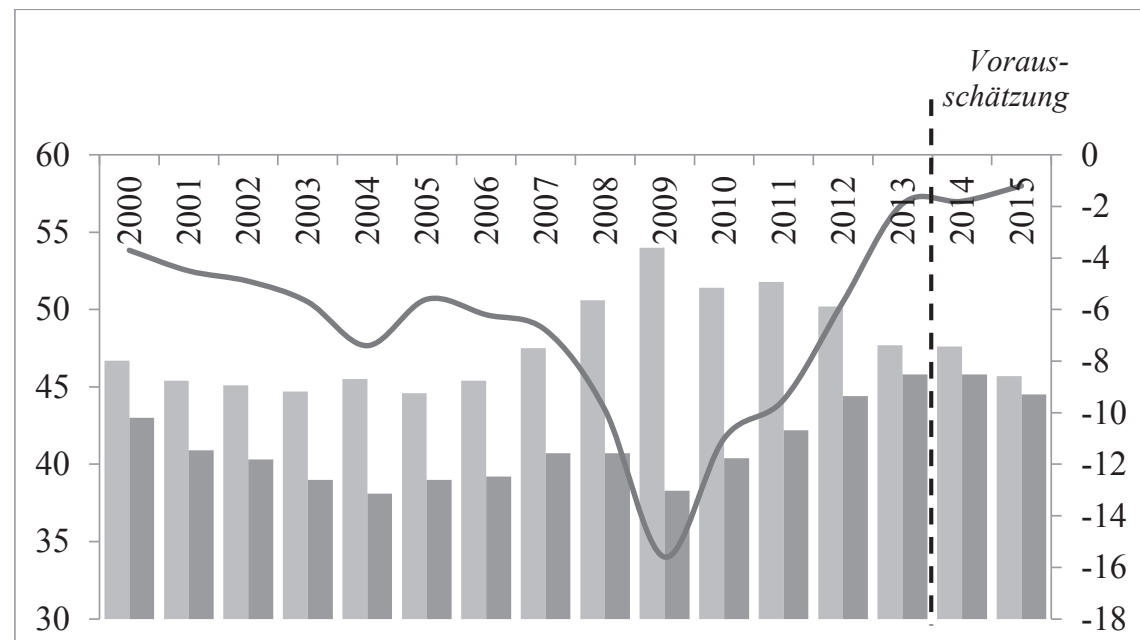

Anmerkung.: Ausgaben für 2011-2013 ohne Kosten für Bankenstützungsmaßnahmen

Quelle: AMECO, Europäische Kommission

\section{Stabilisierung des Finanzsektors}

Im Gegensatz zu anderen Mitgliedstaaten wie Irland, Spanien und Zypern standen Probleme im Bankensektor nicht am Ursprung der Krise in Griechenland. Mit einem Anteil von rund $5 \%$ an ausfallgefährdeten Darlehen zu Beginn des Jahres 2009 standen die griechischen Banken nicht deutlich schlechter da als ihre Konkurrenten in den anderen Mitgliedstaaten der Eurozone. Griechenland hatte keine platzende Immobilienblase, die Verschuldung der Privathaushalte war im Vergleich zu anderen Mitgliedstatten relativ gering und die Banken hatten auf den ersten Blick gesunde Bilanzen. Allerdings bestand nach Expertenmeinung bereits zu diesem Zeitpunkt Bedarf an einer Konsolidierung des Bankensektors (zu hohe Filialdichte, zu starke Marktzersplitterung, etc.).

Die beginnende Rezession und die rapide Verschlechterung der Refinanzierungsmöglichkeiten machten sich jedoch rasch bemerkbar. Seit Ende 2009 hatten 
die griechischen Banken den Zugang zu einer Refinanzierung auf dem Markt verloren und waren zunehmend auf die Unterstützung der EZB angewiesen. Zudem hatte der Bankensektor mit einem dramatischen Rückgang der Spareinlagen zu kämpfen: Zwischen Ende 2009 und Juni 2012, dem Höhepunkt der Krise, sanken die Einlagen um $37 \%$ von rund $237 \mathrm{Mrd}$. Euro auf rund $150 \mathrm{Mrd}$. Euro (Abb. 6).trotz bedeutender Abhebungen.

Zwar haben sich die Einlagen seit ihrem Tiefpunkt wieder etwas erholt, sie liegen mit rund 165 Mrd. im Juni 2014 aber immer noch deutlich unter dem Wert zu Beginn der Wirtschaftskrise.

Abbildung 6: Bankeinlagen (Private Haushalte und Unternehmen)

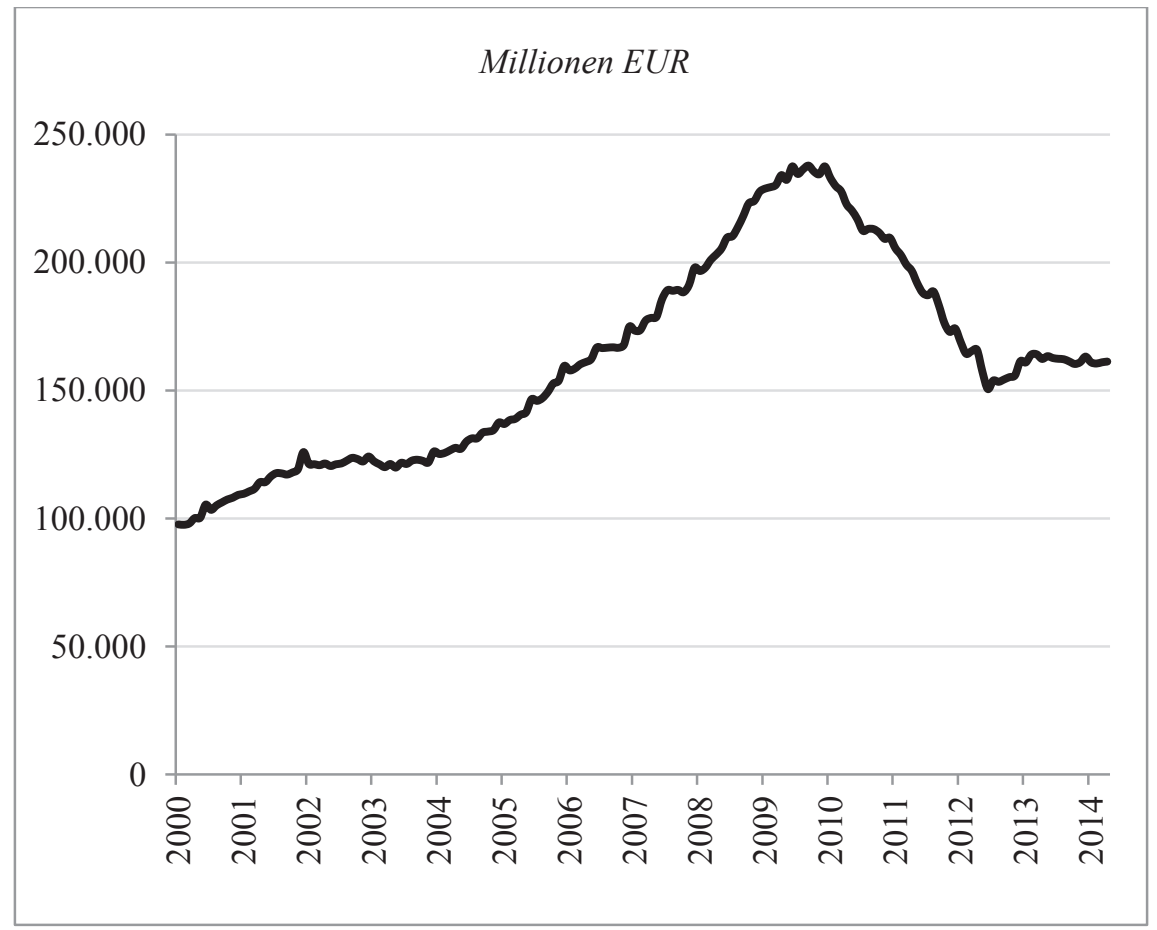

Quelle: Bank of Greece

In der Erwartung einer weiteren Verschlechterung der Lage des Bankensektors hat das erste griechische Anpassungsprogramm die Schaffung eines Fonds für Finanzmarktstabilität (Hellenic Financial Stability Fund - HFSF) mit einer Kapitalausstattung von $10 \mathrm{Mrd}$. Euro vorgesehen. Dies erwies sich jedoch in der Folge als zu gering, denn die griechischen Banken wurden stark von der im Frühjahr 2012 durchgeführten Umschuldung betroffen (siehe III.4). Die vier 
systemischen Banken hatten einen damit verbundenen zusätzlichen Kapitalbedarf von 27,5 Mrd. Euro. ${ }^{7}$ Obwohl ein Teil dieses Kapitals von privaten Investoren aufgebracht wurde, mussten gute $24 \mathrm{Mrd}$. Euro über zusätzliche Mittel des zweiten Anpassungsprogramms eingeschossen werden. Parallel dazu mussten über $14 \mathrm{Mrd}$. Euro der im Programm für Finanzstabilität vorgesehenen $50 \mathrm{Mrd}$. Euro für die Abwicklung kleinerer, nicht überlebensfähiger Banken aufgewendet werden.

Aufgrund der verschlechterten wirtschaftlichen Lage und des starken Anstiegs fauler Kredite stellte die griechische Zentralbank im Februar 2014 einen erneuten zusätzlichen Kapitalbedarf der vier größten Banken von rund $6 \mathrm{Mrd}$. Euro fest. Bei dieser zweiten Kapitalerhöhung konnten diese Großbanken jedoch vom verbesserten Vertrauensklima profitieren und das notwendige Kapital (weitgehend) bei ausländischen Privatinvestoren aufnehmen, sodass keine staatlichen Beihilfen notwendig waren.

Insgesamt hat der Konsolidierungsprozess der letzten Jahre die Bankenlandschaft in Griechenland deutlich verändert. Während der Marktanteil der vier größten Banken zu Beginn der Krise bei rund $72 \%$ lag, stieg er durch die verschiedenen Bankenschließungen bzw. -übernahmen auf fast 98 \% im Frühjahr 2014.

\section{Strukturreformen und Stärkung der Wettbewerbsfähigkeit}

Wie erwähnt, hatte Griechenland beim Eintritt in die Eurozone ein zweistelliges Leistungsbilanzdefizit aufzuweisen. Bereits seit Ende der 90er Jahre stiegen die Lohnstückkosten in Griechenland stärker als im Rest der Eurozone (Abb. 7). Die preisliche Wettbewerbsfähigkeit der griechischen Unternehmen gegenüber den wichtigsten Handelspartnern hatte sich daher bis zu Beginn des Anpassungsprogramms kontinuierlich verschlechtert.

Die Ausarbeitung des Anpassungsprogramms begann mit einer vergleichenden Analyse der Stärken und Schwächen Griechenlands („,benchmarking“). Dabei bestätigte sich, dass Griechenland schwerwiegende Schwachstellen in Bezug auf das Funktionieren der Arbeits- und Gütermärkte hatte.

Was den Arbeitsmarkt betrifft, zeigten sich insbesondere zwei Problemfelder: Erstens beeinträchtigte das hohe Niveau des Beschäftigungsschutzes den Eintritt (oder Wiedereintritt) insbesondere von Jugendlichen, Frauen und Langzeitar-

7 Siehe European Commission: The Second Economic Adjustment Programme for Greece - Third Review, European Economy, Occasional Paper No. 159, Juli 2013, Box 3, 37. 
beitslosen. Zweitens verhinderten die Regeln für die tarifvertragliche Lohnfindung eine Lohnmäßigung und führten zu relativ hohen Mindestlöhnen.

Im Bereich der Güter- und Dienstleistungsmärkte hatte Griechenland nach der Türkei nicht nur die höchste Regulierungsdichte von allen OECD Ländern, ${ }^{8}$ sondern schnitt bei den Indikatoren der Weltbank zum unternehmerischen Umfeld (Unternehmensgründung, Genehmigungsverfahren, usw.) sehr schlecht ab. Gleichzeitig war der Wettbewerb im Verkehrs- wie auch im Energiesektor gering.

In allen diesen Bereichen hat die griechische Regierung im Rahmen des Anpassungsprogrammes Maßnahmen ergriffen, um diese Probleme schrittweise anzugehen. Bei den Arbeitsmarktreformen sind insbesondere Initiativen zur Flexibilisierung der Lohnfindung und zur Reduzierung der Mindestlöhne hervorzuheben sowie die Gesetzgebung hinsichtlich der Regeln zu Entlassungen und Zeitverträgen. ${ }^{9}$

Bei den Reformen der Güter- und Dienstleistungsmärkte reicht die Liste der umgesetzten Reformen von der Liberalisierung des Verkehrssektors über die Öffnung geschlossener Berufe zu Maßnahmen zum Bürokratieabbau und zum Abbau von Beschränkungen des Wettbewerbs in wichtigen Wirtschaftssektoren. Unter Einbeziehung des Schwierigkeitsgrads der Reformen, hat Griechenland nach Einschätzung der OECD in den letzten fünf Jahren stärker auf die Reformempfehlungen reagiert als alle anderen OECD-Länder. ${ }^{10}$ Zudem wurde ein umfangreiches Privatisierungsprogramm von Staatsbetrieben und staatlichen Immobilien in die Wege geleitet, das ursprünglich langfristig Erlöse von bis zu 50 Mrd. Euro erzielt sollte.

8 Siehe $O E C D$ : Economic Surveys: Greece 2013, 33.

9 Für eine Übersicht der wichtigsten Arbeitsmarktreformen seit 2010 siehe European Commission: The Second Economic Adjustment Programme - Fourth Review, European Economy, Occasional Papers, April 2014, 49.

10 Siehe $O E C D$, a.a.O., hier 30. 
Abbildung 7: Nominale Lohnstückkosten

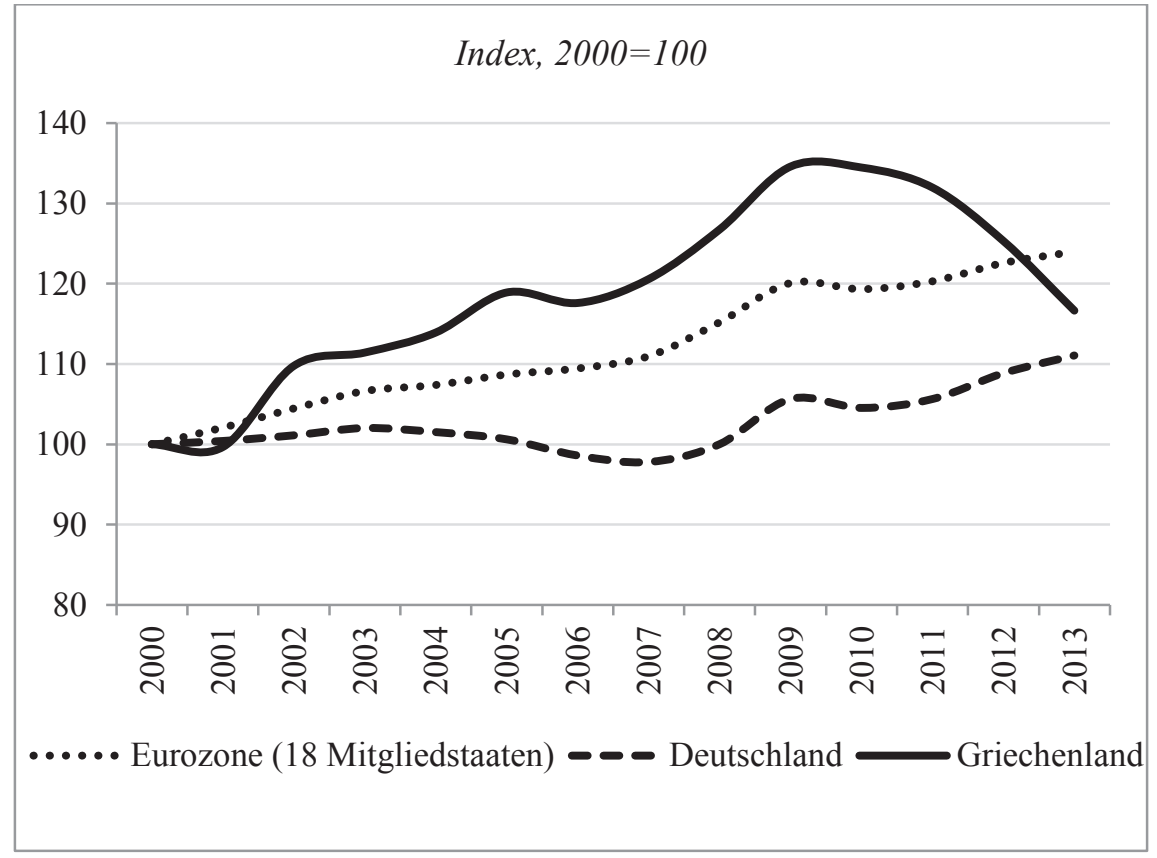

Quelle: Eurostat

\section{Schuldentragfähigkeit}

Gemessen am BIP, wies Griechenland bereits vor Programmbeginn einen der höchsten Schuldenstände in der EU aus (115 \% des BIPs in 2009). Im Rahmen der Krise jedoch kollabierte das Wirtschaftswachstum auch in Griechenland und das Land war seit Ende 2008 in der Rezession. Parallel dazu verschlechterte sich die fiskalische Situation dramatisch (siehe III.1.).

Im Rahmen der Programmverhandlungen musste daher vor dem Hintergrund des negativen Wirtschaftswachstums und der fiskalischen Situation überprüft werden, wie die Schuldentragfähigkeit Griechenlands gesichert werden konnte. Es war außerdem bis zum griechischen Anpassungsprogramm eine der Vorbedingungen des IWF, finanzielle Mittel nur zur Verfügung zu stellen, wenn die Schuldentragfähigkeit mit hoher Wahrscheinlichkeit gesichert ist. Dies war jedoch nach Ansicht der IWF Mitarbeiter nicht der Fall.

Der noch nie dagewesene finanzielle Umfang des Anpassungsprogramms und die bestehenden Zweifel an der Schuldentragfähigkeit führten dazu, dass der IWF erst seine internen Regeln ändern musste bevor er dem Programm zustim- 
men konnte. ${ }^{11}$ Auf Basis der zu Programmbeginn vorliegenden Informationen (einschließlich insbesondere der Sorgen um mögliche systemische Auswirkungen auf den Rest der Eurozone) unterstützte der IWF letztendlich das griechische Anpassungsprogramm. ${ }^{12}$ Die Schuldentragfähigkeitsanalyse der Troika Institutionen deutete allerdings auch auf eine Reihe von bedeutenden Risikofaktoren, die die Schuldentragfähigkeit weiter gefährden könnten. ${ }^{13}$

Mehrere, sich untereinander verstärkende Faktoren trugen dazu bei, dass sich die Situation der Schuldentragfähigkeit nach positivem Programmbeginn zunehmend verschlechterte:

- Größerer Konsolidierungsbedarf: Datenrevisionen erhöhten das tatsächliche Budgetdefizit und erforderten größere fiskalische Anstrengungen als bei Programmbeginn angenommen, um die Programmziele erreichen zu können;

- Tiefere Rezession: Die konjunkturelle Lage verschlechterte sich dramatisch;

- Schwindende politische Stabilität: Die politische Stabilität des Landes und der Regierung verschlechterte sich zusehends und damit die Aneignung des Programms durch griechische Entscheidungsträger;

- Verschlechternde Programmimplementierung: Widerstand gegen die Programmpolitik sowie ungeahnte administrative Kapazitätsprobleme hatten Auswirkungen auf die Programmumsetzung.

Es wurde daher zunehmend deutlicher, dass Griechenland nicht nur zusätzliche Finanzhilfen benötigen würde, sondern auch dass die Dynamik der Verschuldung ohne schuldenreduzierende Maßnahmen nicht unter Kontrolle gebracht werden könnte. Unter den Gläubigerstaaten hatte sich deswegen die Meinung durchgesetzt, private Gläubiger an Stabilisierungsmaßnahmen zu beteiligen („PSI“ Private Sector Involvement). ${ }^{14}$

Die Staats- und Regierungschefs der Eurozone einigten sich daher am 21. Juli 2011 grundsätzlich auf den Finanzrahmen für ein zweites Programm für Grie-

11 Siehe International Monetary Fund: Ex Post Evaluation of Exceptional Access under the 2010 StandBy Agreement, IMF Country Report No. 13/156, Juni 2013, 10.

12 Siehe International Monetary Fund: Greece: Staff Report on Request for Stand-by Arrangement, IMF Country Report No. 10/110, Mai 2010.

13 Siehe Absatz 44 im Kommissionsbericht: European Commission: The Economic Adjustment Programme for Greece -, Occasional Papers 61, Mai 2010, abrufbar unter: http://ec.europa.eu/economy_ finance/publications/occasional_paper/2010/pdf/ocp61_en.pdf.

14 Der Prozess wurde durch die Deutsch-Französische Ërklärung in Deauville vom 18. Oktober 2010 eingeleitet. Siehe für eine kritische seinerzeitige Würdigung der Ereignisse Kaczyński, P.M./ ó Broin, P: From Lisbon to Deauville: Practicalities of the Lisbon Treaty Revision(s), CEPS Policy Brief No. 216, Oktober 2010, abrufbar unter: http://aei.pitt.edu/15132/1/PB_216_Piotr_\%26_Peadar_ on_Lisbon_Revisions.pdf. 
chenland, unter der Maßgabe, dass sich der Privatsektor mit EUR 37 Mrd. daran beteiligen würde, um die Schuldentragfähigkeit zu verbessern. ${ }^{15}$ Es war somit klar, dass die Euroländer nur bereit waren, weitere Finanzmittel zur Verfügung zu stellen, wenn Griechenland eine Schuldenreduzierung mit privaten Gläubigern auf freiwilliger Basis erreichen konnte. Die grundsätzliche Vereinbarung vom Juli 2011 erreichte allerdings nicht die geforderte Mindestbeteiligung von privaten Gläubigern, während sich die Schuldentragfähigkeitssituation weiter verschlechterte.

Vor diesem Hintergrund legten die Staats- und Regierungschefs der Eurozone im Oktober 2011 veränderte Rahmenbedingungen für weitere Finanzhilfen sowie die Einbindung des Privatsektors fest. ${ }^{16} \mathrm{Zu}$ diesem Zweck waren die Eurostaaten bereit, bis zu EUR 30 Mrd. an zusätzlichen Darlehen für den Schuldenumtausch bereitzustellen. Im Gegensatz zur Juli Vereinbarung wurde nicht der Beitrag des Privatsektors als Zielgröße festgelegt, sondern ein Schuldenstand von $120 \%$ des BIPs im Jahre 2020. Nur unter diesen Bedingungen würde der öffentliche Sektor bereit sein, zusätzliche Mittel in Höhe von bis zu EUR 100 Mrd. für ein zweites Programm zur Verfügung zu stellen.

Die monatelangen Verhandlungen zwischen Griechenland und Vertretern der privaten Anleihegläubiger kamen im Februar 2012 zu einem erfolgreichen Abschluss. Am 24. Februar 2012 veröffentlichte Griechenland das Austauschangebot für griechische Anleihen im Wert von rund EUR 206 Mrd. ${ }^{17}$ Das Angebot Griechenlands sah für die privaten Anleihegläubiger im Austausch für bestehende Anleihen vor:

- Neue griechische Anleihen mit einem Nominalwert von 31,5\% des Nominalwertes der ausgetauschten alten Anleihen;

- $\quad$ EFSF Wertpapiere mit einer Laufzeit von 1 oder 2 Jahren mit einem Nominalwert von $15 \%$ des Nominalwertes der ausgetauschten alten Anleihen; und

- Separat handelbare, an die griechische BIP Entwicklung gekoppelte Wertpapiere;

15 Council of the European Union: Statement by the Heads of State or Government of the Euro Area and EU Institutions, Brüssel, 21. Juli 2011, abrufbar unter: http://www.consilium.europa.eu/ uedocs/cms_ data/docs/pressdata/en/ec/123978.pdf.

16 Council of the European Union: Euro Summit Statement, Brüssel, 26. Oktober 2011, abrufbar unter: http://www.consilium.europa.eu/uedocs/cms data/docs/pressdata/en/ec/125644.pdf

17 Für Einzelheiten zur freiwilligen Umschuldüng siehe z.B. Zettelmeyer, J./ Trebesch, Ch./ Gulati, M.: Greek Debt Restructuring: An Autopsy, in: Economic Policy, Juli 2013, 515-563. 
- Griechenland beglich bis zum Austauschdatum aufgelaufene Zinsen durch die Ausgabe kurzfristiger EFSF Wertpapiere.

Insgesamt wurden bis zum Ende der Austauschfrist am 20. April 2012 EUR 198,1 Mrd. von insgesamt EUR 205,6 Mrd. in Frage kommender Anleihen ausgetauscht, was $96 \%$ des Nominalwerts entsprach.

Der Schuldenstand Griechenlands betrug Ende 2011 EUR 355,8 Mrd. oder $165,3 \%$ des BIP. Durch den Anleiheaustausch wurde der Nominalwert des Schuldenstands um rund EUR 105 Mrd. reduziert. Wenn man den revidierten BIP Wert von EUR 195 Mrd. für 2012 heranzieht, entsprach dies einer Reduzierung um rund 54 Prozentpunkte des BIPs. Der Nettoeffekt war allerdings geringer, da der Schuldenumtausch einen Rekapitalisierungsbedarf der heimischen Banken von zusätzlichen EUR 25 Mrd. nach sich zog. Zu Beginn des zweiten Programms wurde der Schuldenstand auf EUR 327 Mrd. oder 160,5\% Ende 2012 geschätzt. Der Schuldenstand sollte nach der damaligen Prognose auf $116,5 \%$ des BIP in 2020 fallen.

Schon bald nach der Entscheidung zum zweiten Programm verschlechterte sich die Situation der Schuldentragfähigkeit erneut deutlich. Dies ist auf eine Reihe von Faktoren zurückzuführen, insbesondere auf die Verschlechterung der makroökonomischen Projektion, einer Verlängerung des Zeitraums für die Defizitrückführung um zwei Jahre, nach unten revidierte Erwartungen zu den Privatisierungserlösen und einer Revision der BIP Zahlen von 2011. Die Troika schätzte daher im Herbst 2012, dass der Schuldenstand bis 2020 nur auf $144 \%$ des BIP fallen würde, sollten keine weiteren Maßnahmen ergriffen werden.

Vor diesem Hintergrund verhandelten die Euroländer mit Griechenland eine Reihe von Sondermaßnahmen, um den Schuldenstand auf revidierte Zielgrößen von $124 \%$ des BIP in 2020 und deutlich unter $110 \%$ des BIP in $2024 \mathrm{zu}$ bringen. ${ }^{18}$ Insbesondere führte Griechenland Anfang Dezember 2012 eine Schuldenrückkaufsoperation durch, um von dem merklichen Abschlag zu profitieren, mit dem die neuen griechischen Anleihen in den Märkten gehandelt wurden. Vor dem Schuldenrückkauf belief sich die Gesamtsumme der neuen griechischen Anleihen auf EUR 62 Mrd. An dem Rückkaufsangebot nahmen rund EUR 31,9 Mrd. zu einem Durchschnittspreis von 33,8\% des Nominalwerts teil. Damit wurde der griechische Schuldenstand netto um EUR 21,1 Mrd. gesenkt.

18 Siehe Absatz 110 des Kommissionsberichts für mehr Details. European Commission: The Second Economic Adjustment Programme for Greece - First Review, European Economy, Occasional Paper No. 123, Dezember 2011, abrufbar unter: http://ec.europa.eu/economy_finance/publications/ occasional_paper/2012/pdf/ocp123_en.pdf. 
Gleichzeitig haben die Mitglieder der Eurozone im November 2012 zugesagt, in Erwägung zu ziehen, Griechenland weiter Hilfen und Unterstützung zukommen zu lassen, falls dies zum Erreichen der Schuldenreduktionsziele notwendig ist, vorausgesetzt Griechenland respektiert die Haushaltskonsolidierungsvorgaben und die anderen Bedingungen des wirtschaftlichen Anpassungsprogrammes.

Die politischen Diskussionen über mögliche weiter Unterstützungsmaßnahmen werden voraussichtlich im Spätherbst 2014 stattfinden sofern die im September begonnene regelmäßige Überprüfung der Fortschritte bei der Umsetzung des Anpassungsprogramms zu einem positiven Ergebnis kommt. Dabei wird es zunächst darum gehen, den Finanzbedarf Griechenlands in den Jahren 2015/2016 und seine mögliche Deckung zu analysieren, da das laufende EFSF-finanzierte Hilfsprogramm Ende 2014 ausläuft. Zum anderen wird sich anhand einer auf den neuesten Stand gebrachten Schuldentragfähigkeitsanalyse die Frage stellen, ob und gegebenenfalls welche -weitere Maßnahmen zur Schuldenreduzierung angebracht sind.

\section{Einschätzung des wirtschaftlichen Anpassungsprogramms und Aus- blick}

Für eine abschließende Bewertung des Anpassungsprogramms ist es noch zu früh und ein objektiver Erfolgsmaßstab - abgesehen vom Vermeiden des Auseinanderbrechens der Eurozone oder eines Austrittes Griechenlands aus der Eurozone - ist nicht einfach zu definieren. Einige vorläufige Einschätzungen sind jedoch bereits zum gegenwärtigen Zeitpunkt möglich. Das griechische Anpassungsprogramm ist von vielen Seiten für eine übertrieben rasche Haushaltskonsolidierung, einen unterlassenen Schuldenschnitt zu Programmbeginn sowie schwere Prognosefehler kritisiert worden. ${ }^{19}$

Die Frage ist legitim, ob im ersten Anpassungsprogramm ein größerer Schwerpunkt auf Strukturreformen hätte gelegt werden sollen und im Gegenzug das Konsolidierungstempo hätte verringert werden sollen. Wie auch in anderen Ländern der Eurozone sichtbar, ist es für jede Regierung schwierig, politisches Kapital gleichzeitig für einschneidende Konsolidierungsmaßnahmen und unpopuläre Strukturreformen aufzubringen und die dafür notwendigen parlamentarischen Mehrheiten zu sichern. Angesichts der dramatischen fiskalischen Lage zu Beginn des Jahres 2010 stand zwangsläufig das Ziel einer fiskalischen Stabilisierung der Lage im Zentrum der kurzfristigen Bemühungen. Die Wiederherstellung der

19 Siehe hierzu auch. International Monetary Fund: Ex-Post Evaluation, a.a.O., hier 26ff. 
internationalen Wettbewerbsfähigkeit und der Neuorientierung der Volkswirtschaft waren jedoch von Beginn an das mittelfristige Programmziel. Es ist auch zu berücksichtigen, dass bei den anderen Mitgliedstaaten der Eurozone anlässlich eines für viele überraschenden zweistelligen Haushaltsdefizits und des Gefühls Griechenland hätte sich den Eintritt in die Eurozone durch manipulierte Statistiken ,erschlichen“, wenig politische Bereitschaft zu erkennen war, dem Land viel Zeit zum Erreichen solider Finanzen zu geben. Eine verlangsamte Haushaltskonsolidierung hätte zudem den historisch bereits außergewöhnlich hohen Programmfinanzierungsbedarf noch weiter erhöht.

Die Troika wurde in diesem Zusammenanhang auch dafür kritisiert, die makroökonomischen Auswirkungen einer forcierten Haushaltskonsolidierung unterschätzt zu haben. Es lässt sich nicht leugnen, dass Prognosen in Zeiten schwerer Wirtschaftskrisen mit besonderer Unsicherheit behaftet sind. Zudem war die notwendige ,interne Abwertung“ zur Wiederherstellung der preislichen Wettbewerbsfähigkeit innerhalb der Währungsunion in mancher Hinsicht Neuland für Anpassungsprogramme. Die Debatte um ,unterschätzte Multiplikatoren“20 vernachlässigt aber zwei wichtige Faktoren. Zum einen ist bekannt, dass eine Haushaltskonsolidierung mit größeren volkswirtschaftlichen Kosten verbunden ist, wenn sie als nicht glaubhaft eingeschätzt wird. Viele Beobachter bezweifelten unstreitig lange, dass Griechenland zu einer raschen Haushaltskonsolidierung willens und fähig wäre. Zum anderen lassen sich Situationen wie in Griechenland mit weitverbreiteten Spekulationen über einen imminenten Staatsbankrott sowie einen Austritt aus der Eurozone sicherlich nicht mit einem traditionellen Multiplikatoransatz angemessen erfassen. Im Gegenteil, eine expansive Fiskalpolitik hätte diese „Grexit“ Spekulationen zweifellos nur befördert und daher vermutlich einen negativen Multiplikator gehabt.

Die weitverbreiteten Zweifel an der Anpassungsfähigkeit Griechenlands hatten einen deutlichen Einfluss auf das Anpassungsprogramm. Anders als in Irland und Portugal gab es zu Beginn des Anpassungsprogramms keinen parteiübergreifenden Konsens zur Notwendigkeit drastischer Anpassungsmaßnahmen. Obwohl die Regierung ihren Reformwillen überzeugend darlegte, unterschätzte die Troika zu Beginn des Anpassungsprogramms die Widerstände einflussreicher Interessengruppen einerseits und überschätzte die Kapazität der öffentlichen Verwaltung andererseits, ein anspruchsvolles Anpassungsprogramm zügig zu entwickeln und umzusetzen. Die fehlende Aneignung des Reformprogramms 
durch weite Teile der Verwaltung führte in der Folge zu immer längeren und detaillierteren Memoranda zur Programmkonditionalität. ${ }^{21}$ Die Tatsache, dass ein zweites Anpassungsprogramm notwendig war, zeigt, dass alle Beteiligten im Frühjahr 2010 die Schwere der Aufgabe deutlich unterschätzt haben.

Viele Beobachter, ${ }^{22}$ auch innerhalb des IWF, ${ }^{23}$ vertreten die Ansicht, es hätte zu einem früheren Zeitpunkt, sogar zu Beginn des Programms, einen drastischen Schuldenschnitt geben sollen. Bei der Einschätzung dieser Frage sind jedoch zwei wichtige Punkte zu beachten. Erstens war die Eurozone zu diesem Zeitpunkt in keiner Weise auf einen derartigen Schritt vorbereitet. Es herrschte die Furcht, ein Schuldenschnitt für Griechenland würde zu Spekulationen über eine vergleichbare Maßnahme für andere Mitgliedstaaten, wie z.B. Portugal oder Italien, führen. Für Italien hätte dies zu einer weiteren Verschlechterung seiner Schuldenfinanzierungsbedingungen geführt. Bei der damaligen Fragilität und Volatilität der Finanzmärkte stellte dies ein unvertretbares systemisches Risiko dar. Zum zweiten hätte ein vermeintlich schmerzloser Schuldenschnitt die Konsolidierungs- und Reformanreize für Griechenland (und möglicherweise andere EU Mitgliedsstaaten) negativ beeinflusst („moral hazard“). Zu einem Zeitpunkt als Viele in Griechenland das Anpassungsprogramm bekämpften, hätte die Gefahr bestanden, dass ein Schuldenschnitt zwar kurzfristig die Lage verbessert, die schmerzhaften strukturellen Reformen daher jedoch nicht umgesetzt worden wären. Tatsächlich wären keine der tiefliegenden strukturellen Probleme Griechenlands mit einem Schuldenschnitt beseitigt gewesen.

Aus heutiger Sicht und angesichts der tiefgreifenden Missstände in praktisch allen Bereichen von Wirtschaft und Verwaltung zu Beginn des Anpassungsprogramms, ist Griechenland bei der Erreichung der Ziele des Anpassungsprogramms weit vorangekommen. Konjunkturbereinigt hat Griechenland einen der höchsten strukturellen Haushaltsüberschüsse in der EU. Gleichzeitig sind die Lohnstückkosten deutlich gesunken (Abb. 7). Damit hat sich die kostenmäßige Wettbewerbsfähigkeit und, wenn auch verzögert und nicht in gleichem Umfang, auch die preisliche Wettbewerbsfähigkeit verbessert. Zudem hat Griechenland nach Einschätzung der OECD in den letzten fünf Jahren mehr Strukturreformen umgesetzt als alle anderen OECD Länder. Der Bankensektor ist stabilisiert und

21 Alleine für das Jahr 2013 sahen die jeweiligen Memoranda die Umsetzung von fast 900 verschiedenen Einzelmaßnahmen vor.

22 Siehe z.B. Pisani-Ferry, J./ Sapir, A./ Wolff, G.: EU-IMF assistance to euro-area countries: an early assessment, Bruegel, Brussels, 2013, 65ff.

23 Siehe International Monetary Fund: Ex-Post Evaluation, a.a.O., 26. 
das Vertrauen ausländischer Investoren in Griechenland hat sich sehr deutlich verbessert.

Die Schattenseite dieses Anpassungserfolges sind Einbußen des verfügbaren Einkommens um über $30 \%$ seit Beginn der Krise und eine Arbeitslosenquote von rund 27 \%. Für das Jahr 2014 erwartet die Troika jedoch ein Ende der Rezession und für die nächsten Jahre ein kräftiges Wirtschaftswachstum in der Größenordnung von rund $3 \%$ (Abb. 1). Die zahlreichen Strukturreformen der letzten Jahre sollten dieses Wachstum auf eine gesunde Grundlage stellen.

Trotz der unbestreitbaren Erfolge hat Griechenland in vielen Bereichen noch wichtige Aufgaben vor sich. Im Haushaltsbereich hat das Land zwar (in der Programmdefinition) 2013 einen Primärüberschuss von 0,8\% des BIP erreicht. Das Programmziel eines Primärüberschusses von 4,5\% für 2016 wird jedoch trotz des erwarteten kräftigen Wirtschaftswachstums weiter Konsolidierungsanstrengungen erfordern. Auch ist die Reform der Steuerverwaltung bei weitem noch nicht abgeschlossen. Gleichzeitig belastet der hohe Bestand an ausfallgefährdeten Krediten den Bankensektor und beschränkt die Kreditvergabe.

Auch im Bereich der Strukturreformen ist noch erheblicher Reformbedarf. Trotz der umfassenden Reformen der letzten Jahre hat Griechenland immer noch ein überdurchschnittlich hohes Regulierungsniveau von Güter- und Dienstleistungsmärkten. ${ }^{24}$ Zudem stand es Ende 2013 lediglich auf Platz 72 der Weltbank Rangliste $\mathrm{zu}$ den unternehmerischen Rahmenbedingungen, mit am schlechtesten von allen EU-Mitgliedstaaten. ${ }^{25}$ Obwohl sich die preisliche Wettbewerbsfähigkeit deutlich verbessert hat, sind weitere tiefgreifende strukturelle und institutionelle Reformen notwendig um das Ausfuhrpotential der griechischen Volkswirtschaft auf das Niveau anderer EU-Mitgliedstaaten zu bringen. ${ }^{26}$ Es wird sich zeigen, ob nach mehr als vier Jahren Anpassungsprogramm noch der politische Wille und die parlamentarischen Mehrheiten für weitergehende Strukturreformen vorhanden sind.

Siehe OECD: Economic Policy Reform 2014: Going for Growth, Interim Report, 2014, 69

Siehe World Bank: Doing Business 2014, Economic Profile: Greece, 2014.

Siehe z.B. Böwer, U./ Michou, V/ Ungerer, Ch.: The Puzzle of the Missing Greek Exports, European Commission, Economic Papers No. 518, 2014. 


\section{Die Politik und Zusammenarbeit der Troika Partner}

\section{Beweggründe zur Entstehung}

Die Zusammenarbeit von IWF und der Europäischen Kommission hatte nicht erst mit dem griechischen Programm begonnen. Vor Griechenland gab es die gemeinsam finanzierten Anpassungsprogramme in Ungarn, Lettland und Rumänien. Die EZB war bereits im Falle Lettlands wegen des „currency boards“ eingebunden. Die eigentliche Troika Konfiguration mit Kommission, EZB und IWF startete jedoch erst mit dem griechischen Programm, gefolgt von den Programmen für Irland, Portugal und Zypern.

Die Kommission brachte in die Troika ihre langjährige Erfahrung zu makroökonomischen, fiskalischen und strukturpolitischen Politiken ein, die sie im Rahmen der länderspezifischen Überwachung gewonnen hatte. Zudem ist die Kommission insgesamt für die Einhaltung der vertraglichen Verpflichtungen (sogenannter „acquis communautaire“) der Mitgliedsstaaten verantwortlich.

Eine Reihe von Gründen sprach für die Einbindung von IWF und EZB:

- Die Nutzung der langjährigen Erfahrung und Kompetenz des IWF mit Anpassungsprogrammen (Gestaltung und Überwachung);

- Die Überzeugung der Mitgliedsstaaten, dass die Einbindung des IWF und der EZB die Glaubwürdigkeit der Programmgestaltung und -überwachung erhöht;

- Die Einschätzung dass der IWF weniger politischen Zwängen unterliegt als die Europäische Kommission;

- Die anfängliche Abwesenheit von angemessenen Hilfsinstrumenten für Euroländer;

- Die globale Rolle des IWF bei der Bereitstellung von externer Finanzhilfe;

- Die wichtige Rolle der EZB bei der Liquiditätsversorgung der Banken in den Programmländern;

- Die besondere Expertise der EZB zu finanzsektorspezifischen und monetären Fragen.

Nichtsdestotrotz gab es im Vorlauf zu den Diskussionen um Griechenland auch abweichende Meinungen. Gerade Deutschland vertrat anfänglich die Meinung, dass die Eurozone alleine ihre Probleme meistern müsste und nicht auf den IWF zugreifen sollte. Nach einiger Zeit schwenkte Deutschland jedoch um und befürwortete vehement eine Einbindung und Beteiligung des IWF an den Programmen in Euroländern. 


\section{Eine Beschreibung der operativen Zusammenarbeit}

Die Troika Teams setzen sich in ihrer Struktur ähnlich zusammen und sind auch in allen Anpassungsprogrammen vergleichbar. Sie werden von hochrangigen Funktionären geleitet („Head of Missions“), die von einem Stellvertreter und einer Reihe von Experten unterstützt werden. Im Wesentlichen decken die Experten der Teams die Bereiche makroökonomische Analysen, Fiskalpolitik, Finanzmärkte, Programmfinanzierung ${ }^{27}$ und strukturpolitische Themen (z.B. Privatisierung, Arbeitsmärkte, Gesundheitssektor, Transport und Energie etc.) ab.

Die Größe der Teams variiert; in der Regel jedoch ist das Kommissionsteam das umfangreichste, da die Kommission aufgrund ihrer Zuständigkeit als „Hüterin der Verträge" alle im Memorandum enthaltenen Politikfelder abdeckt und die Einhaltung der damit zusammenhängenden Konditionalität überwacht, während sich IWF und EZB im Einklang mit dem jeweiligen institutionellen Mandat auf eine begrenztere Anzahl von Themenfeldern konzentrieren können.

Die jeweiligen Experten aus den verschiedenen Teams arbeiten zu den entsprechenden Themen eng zusammen; so werden beispielsweise Sitzungen und Besprechungen zu einem bestimmten Themenkomplex von den Experten der Teams gemeinsam organisiert und abgehalten oder Anfragen an die nationalen Behörden gemeinsam vorgebracht. Der Austausch zwischen den Troika Teams ist nicht auf die Missionen vor Ort beschränkt, sondern findet kontinuierlich zwischen den Länderteams per E-Mail, Telefon oder Videokonferenz statt. Abgesehen von wenigen speziellen Themen, die nicht alle Troika Partner betreffen, werden die Informationen, die die nationalen Autoritäten zur Verfügung stellen, zwischen allen Troika Partnern geteilt. Projektionen, Reaktionen, Analysen und Kommentare zu Konditionalität sowie zu Vorschlägen und Daten aus den betroffenen Ländern werden in den meisten Fällen zwischen den Troika Partnern ausführlich diskutiert, abgestimmt und als gemeinsame Position gegenüber den nationalen Behörden kommuniziert.

Die Programmarbeit war für die Kommission und die EZB in gewisser Weise Neuland. Trotz erster Erfahrungen im Rahmen der Programme für die NichtEuroländer, stellte das griechische Programm in vielfacher Hinsicht eine völlig neue Dimension dar: Umfang der Hilfe, makroökonomischer und strukturpolitischer Anpassungsbedarf sowie die politische Situation im Land. Und im Gegen-

27 Wenn Themen der Programmfinanzierung diskutiert werden, sind auch Vertreter des EFSF involviert, da der EFSF die EU Auszahlungen auf den Märkten refinanzieren muss und deshalb früh eingebunden werden muss. 
satz zu den Programmen der Nicht-Euroländer Ungarn und Rumänien, wo der Großteil der Finanzierung vom IWF kam, stellte die EU den überwiegenden Teil der Finanzhilfen an die Euroländer bereit.

Es fiel dem IWF anfänglich nicht immer leicht, die daraus erwachsende informelle Führungsrolle der Kommission zu akzeptieren. Bis zum Beginn des Griechenland Programms hatte der IWF in der Programmarbeit in der Regel zumindest die Führungsposition innegehabt oder konnte sogar ganz unabhängig agieren. Dieser Gewöhnungsprozess zeigte sich in mehreren Bereichen wie der Absprache der Troika Partner auf gemeinsame Verhandlungslinien, der zur Verfügungstellung von internen Dokumenten vor den Aufenthalten vor Ort und der Kommunikationspolitik nach außen.

Auf Seiten der EZB bestand die Herausforderung, wichtige Daten und Informationen in die Programmanalyse der Troika einzuarbeiten, die der EZB aufgrund ihrer Funktion als Zentralbank der Eurozone zur Verfügung gestellt wurden, ohne den marktsensiblen und hochvertraulichen Charakter der Daten zu verletzen.

Insgesamt jedoch hat die Zusammenarbeit der Troika Teams auf technischer Ebene sowohl vor Ort wie zwischen den Aufenthalten vor Ort gut funktioniert. Gelegentliche Spannungen zwischen den Institutionen konnten in der Regel relativ schnell beigelegt werden und die technischen Teams stimmten in ihren Einschätzungen häufig überein.

Die Entscheidungsabläufe auf europäischer Ebene und im IWF sind jedoch nach wie vor getrennt. Auf europäischer Seite werden alle wichtigen Programmentscheidungen von der Eurogruppe (das Gremium der Finanzminister der Mitgliedstaaten der Eurozone) getroffen. Entscheidungen der Eurogruppe (und des EFSF/ESM Direktoriums) über die Auszahlung einer weiteren Tranche des Darlehens werden auf der Grundlage eines in Verbindung mit der EZB erstellten Berichts der Kommissionsdienststellen getroffen. Auf Seite des IWF werden derartige Entscheidungen vom „Executive Board“ auf Grundlage eines Berichtes der IWF Mitarbeiter getroffen. Es gibt also formal gesehen keinen gemeinsamen „Troika-Bericht“, auch wenn die jeweiligen Berichte weitgehend abgestimmt sind.

\section{Eine Diskussion der Vor- und Nachteile: Eine kritische Betrachtung}

Die Zusammenarbeit der drei Institutionen im Rahmen der Troika war ein Lernprozess für alle Beteiligten. Es brauchte eine gewisse Zeit, gegenseitiges Ver- 
ständnis für die unterschiedlichen Rollen, Mandate und institutionellen Beschränkungen zu entwickeln. IWF Mitarbeiter waren anfänglich nicht mit dem rechtlichen und regulatorischen Rahmenwerk der EU vertraut sowie mit den daraus folgenden Zwängen für die Kommission. ${ }^{28}$ Kommissionsmitarbeiter hatten ihrerseits keine profunde Kenntnis des internen IWF Regelwerks (z.B. Bedingungen für den Zugang zu Finanzmitteln, Schuldentragfähigkeitsanalysen etc.).

Die Herangehensweise der Institutionen und der den Institutionen zur Verfügung stehende Ermessensspielraum haben sich deutlich unterschieden und spiegeln die spezifischen Institutionskulturen, Erfahrungsschatz, Kompetenzen und Mandate wider:

- Das IWF Mandat ist stark auf fiskalische Ziele und einige zentrale Strukturreformen fokussiert. Die IWF Mitarbeiter arbeiten im Interesse ihrer Institution und dessen eigener Finanzmittel. Die Programmarbeit des IWF basiert auf einem über die Jahrzehnte entwickelten Bestand an ökonomischen Analysen und internen Regeln. ${ }^{29}$ Dieses Regelwerk erlaubt es den IWF Mitarbeitern vor Ort, innerhalb ihres Verhandlungsmandats relativ unabhängig vom IWF Board zu agieren. Das IWF Board ist auch nicht in das spezifische Verhandlungsmandat involviert. Allerdings beruht der langjährige IWF Erfahrungsschatz und Doktrin im Wesentlichen auf Programmen in individuellen, unabhängigen und weniger entwickelten Ländern, die nicht ohne weiteres auf eine entwickelte Volkswirtschaft übertragbar sind, die Mitglied einer politischen und Währungsunion ist.

- Das Kommissionsmandat dagegen ist viel breiter und gründet sich auf dem Respekt der Verpflichtungen des EU Vertrags und des gemeinschaftlichen Gesetzesbestands. So muss Programmkonditionalität zum Beispiel im Einklang mit europäischen Regeln zu Staatsbeihilfen sein. Gleichzeitig ist die Integrität der Eurozone ein übergreifendes politisches Ziel. Im Falle der Euroländer verhandeln die Kommissionsmitarbeiter zum Großteil - im Falle Griechenland ausschließlich - über Finanzmittel, die von den Euroländern zur Verfügung gestellt wurden und nicht über gemeinschaftliche Mittel. ${ }^{30}$ Die Kommissionsvertreter verhandeln also nicht auf der Grundlage einer

So kann die Kommission keine Vorschläge für Maßnahmen mittragen, die nicht im Einklang mit den EU Vertragen sind.

29 So verlangen die internen IWF Regeln beispielsweise, dass ein Programm 12 Monate voraus voll finanziert sein muss (,12-month financing assurances“), um IWF Mittel auszuzahlen. Oder es gibt besondere Anforderungen, wenn die angefragten IWF Mittel $200 \%$ der Anteilsquote des Landes beim IWF übersteigen (,exceptional access procedure“).

30 Der europäische Anteil am ersten Programm wurde über bilaterale Darlehen der Euroländer an Griechenland zur Verfügung gestellt, die in der sogenannten „Griechischen Darlehensfazilität" gepoolt wurden. Das zweite Programm wird über die Europäische Finanzstabilitätsfazilität (EFSF) finanziert, die von den Garantien der Euroländer getragen wird. 
Gemeinschaftskompetenz, sondern im Auftrag der Mitgliedstaaten der Eurozone. Der Vorsitzende der Eurogruppe-Arbeitsgruppe (EAG) ist daher in die Ausarbeitung des Verhandlungsmandats vor Beginn der Verhandlungen eingebunden und die Kommissionsvertreter müssen die EAG und Eurogruppe auch während der Verhandlungen auf dem Laufenden halten. Die Euroländer haben zum Beispiel bei den Verhandlungen über ein Programm mit Zypern frühzeitig einen maximalen Finanzierungsrahmen festgelegt, was erhebliche Auswirkungen auf die Verhandlungen und den Programmzuschnitt hatte.

- Obwohl das Kernmandat der EZB im Prinzip deutlich enger gefasst ist als der Programmzuschnitt, haben die Mitgliedstaaten der Eurozone Wert auf die Ratschläge der EZB zu allen Programmbereichen gelegt. Von mancher Seite wird auf mögliche Interessenkonflikte der EZB verwiesen. ${ }^{31}$ Dem kann aber auch entgegengestellt werden, dass die EZB mit ihren Vorschlägen zur Haushaltskonsolidierung, Strukturreformen und Maßnahmen zur Sicherung der Finanzstabilität indirekt auch den Transmissionsmechanismus der Geldpolitik in den betreffenden Mitgliedstaaten wiederherzustellen hilft.

In vielen Bereichen stimmten die Analysen und Einschätzungen der Troika Partner überein. Gerade im Falle Griechenlands gab es jedoch eine Reihe von Themen, die relativ kontrovers und über einen längeren Zeitpunkt diskutiert wurden. Im Extremfall drangen diese anfänglichen Unstimmigkeiten auch nach außen, wenn es um Angelegenheiten von fundamentaler Bedeutung ging (z.B. Schuldentragfähigkeit). Dabei handelte es sich meist um Angelegenheiten, bei denen noch kein Konsensus zwischen den Euroländern bestand und die Troika Institutionen um ihre unterschiedlichen Vorschläge auf verschiedenen Wegen warben. Diese Spannungen haben jedoch die Entwicklung neuer Ideen durch die Troika Teams und ihre jeweiligen komparativen Wissensvorteile stimuliert und Unstimmigkeiten konnten letztlich beigelegt werden. Alles in allem vermochten die Troika Institutionen in der großen Mehrheit der Themen, sich auf gemeinsame Positionen zu einigen, bevor diese mit den Euroländern diskutiert werden.

\section{Zusammenfassende Einschätzung des Troika-Ansatzes und Ausblick}

Insgesamt gesehen, kann die Zusammenarbeit innerhalb der Troika im Rahmen der Anpassungsprogramme für Griechenland, Irland, Portugal und Zypern als erfolgreich angesehen werden. Die Zusammenführung der jeweiligen Erfahrung und Expertise des IWF, der EZB und der Europäische Kommission ist der Quali-

31 Siehe z.B. Pisani-Ferry, J./Sapir, A./ Wolff, G., a.a.O., hier 111. 
tät der Analyse und der Programmempfehlungen zugutegekommen auch wenn sie einen höheren Koordinierungsbedarf impliziert.

Es ist jedoch einzuräumen, dass wichtige Strategieunterschiede zwischen den drei beteiligten Institutionen die Zusammenarbeit innerhalb der Troika zeitweise erschwert und zum Teil langwierige Diskussionen auf politischer Ebene (insbesondere innerhalb der Eurogruppe der Finanzminister) erfordert haben. Beispiele hierfür sind die langwierigen Diskussionen über die Schuldentragfähigkeit Griechenlands und Zyperns.

Das Europäische Parlament (EP) hat sich im Februar 2014 in einem Bericht kritisch mit der Rolle und den Tätigkeiten der Troika in Programmländern des Euroraums auseinandergesetzt. ${ }^{32}$ In einer Entschließung vom 13. März 2014 wird festgestellt, dass das Mandat der Troika aufgrund der ständigen Weiterentwicklung der Reaktion der Europäischen Union auf die Krise, der unklaren Rolle der EZB in der Troika und der Art des Entscheidungsprozesses der Troika als unklar, intransparent und einer demokratischen Kontrolle entbehrend wahrgenommen wurde. ${ }^{33}$ Dazu ist anzumerken, dass der EFSF kein Gemeinschaftsinstrument, sondern ein intergouvernementales Konstrukt ist und die einzelnen Mitgliedstaaten daher als Anteilseigener des EFSF der Kontrolle ihrer nationalen Parlamente unterlagen. Zudem stand der für Wirtschaft und Finanzen zuständige Vizepräsident der Kommission dem Europäischen Parlament regelmäßig für einen Meinungsaustausch zur Verfügung.

Das Parlament weist auf mögliche Interessenkonflikte der EZB hin und hinterfragt auch die Doppelrolle der Europäischen Kommission als Vertreterin der Mitgliedstaaten des Euroraums einerseits und als Gemeinschaftsorgan andererseits. Mittel- und langfristig fordert das EP die Schaffung eines Europäischen Währungsfonds auf der Grundlage des Unionsrechts.

$\mathrm{Ob}$ das Troika Modell auch bei möglichen zukünftigen Programmen innerhalb der Eurozone Anwendung finden sollte, bedarf einer gründlichen Diskussion. Im Vergleich zur Situation 2010 hat sich die Ausgangslage in einer Reihe von Punkten geändert. Die Europäische Kommission hat in den letzten Jahren zusätzliche Programmerfahrung gesammelt; die EZB hat im Bereich der Finanzaufsicht weitreichende neue Kompetenzen erhalten welche Fragen eines möglichen Inte-

32 Europäisches Parlament: Bericht über die Rolle und die Tätigkeiten der Troika (EZB, Kommission und IWF) in Bezug auf Programmländer des Euroraums, A7-0149/2014, 2014.

33 Europäisches Parlament, Entschließung über die Rolle und Tätigkeiten der Troika in Bezug auf Programmländer des Euroraums, P7_TA 0239, 2014. 
ressenkonflikts aufwerfen könnten; innerhalb des IWF gibt es zunehmende Bedenken von außereuropäischen Staaten gegen ein weiteres Engagement in der Eurozone und nicht zuletzt ist mit dem Europäischen Stabilitätsmechanismus (ESM) ein zusätzlicher Teilnehmer geschaffen worden. Von manchen wird daher zur Vereinfachung die Schaffung eines Europäischen Wirtschaftsfonds (EWF) vorgeschlagen. ${ }^{34}$ Nach seiner Nominierung als zukünftiger Präsident der Europäischen Kommission hat Jean-Claude Juncker am 15. Juli in seinen politischen Leitlinien für die Amtszeit der nächsten Kommission vorgeschlagen, die Troika in Zukunft durch eine stärker demokratisch legitimierte und rechenschaftspflichtige Struktur zu ersetzen, auf der Grundlage europäischer Institutionen mit einer besseren parlamentarischen Kontrolle sowohl auf europäischer als auch auf nationaler Ebene. ${ }^{35}$ Lösungen, welche eine Änderung der Verträge zur Europäischen Union erfordern, können aber realistischerweise nur mittel- bis langfristig umgesetzt werden. Bis dahin bleibt zu hoffen, dass keine weiteren Anpassungsprogramme von Nöten sind. 